\title{
Arenc : le premier centre de rétention était clandestin
}

\author{
Ed Naylor ${ }^{1}$, University of Portsmouth (Royaume-Uni)
}

On lit sur le site du ministère de l'intérieur que «les centres de rétention administrative ont été officiellement créés par la loi du 29 octobre $1981 »{ }^{2}$ Derrière cette phrase c'est toute l'histoire du centre d'Arenc, un hangar des quais du port de Marseille où, de 1963 à 1975, des milliers d'étrangers ont été détenus, à la discrétion de l'administration, jusqu'à leur embarquement forcé sur un navire. Ce centre de rétention « avant la lettre ${ }^{3}$ » existait en dehors de tout cadre juridique, les détenus étaient privés d'accès à un avocat et de tout contact avec l'extérieur. En 1975, l'enquête d'un avocat et d'un journaliste dénonce publiquement cette «prison clandestine ». «L'affaire d'Arenc» était lancée et, dans la foulée, deux anciens détenus portaient plainte contre X pour séquestration : la controverse politique enfla et atteignit les bancs de l'Assemblée nationale.

\section{L'ouverture d'Arenc}

Le centre occupait le deuxième étage d'un hangar désaffecté d'une zone industrielle à proximité de la gare maritime. Ces locaux (quelques bureaux et une salle d'attente, accessibles uniquement par un escalier métallique externe) avaient été cédés à la Sécurité publique par la Chambre de commerce de Marseille au début des années 1960. L'utilisation de ce bâtiment comme lieu de détention se situe dans le contexte de la fin de la Guerre d'indépendance algérienne (1954-1962). Les accords d'Evian de mars 1962 prévoyaient un régime de « libre circulation » entre les deux pays. Pour le gouvernement français il s'agissait surtout d'obtenir des garanties sur l'avenir de la population européenne dans une Algérie indépendante, dans l'espoir d'éviter un afflux massif vers la métropole. Or, entre avril et septembre 1962, la grande majorité des « pieds-noirs » traversent la Méditerranée. La réciprocité rendue ainsi caduque, le gouvernement français cherche à renégocier les conditions de circulation entre les deux pays. Dans un rapport d'octobre 1962, les conseillers techniques aux affaires musulmanes (CTAM) de Marseille alertent sur « l'accélération anarchique du mouvement migratoire des Musulmans algériens dans le sens Algérie-France », et, confirmant l'analyse de la

\footnotetext{
1 Je tiens à remercier mes collaborateurs du projet "Lieux à mémoires multiples et enjeux d'interculturalités" (LMM) soutenu par le ministère de la culture. Je suis également très reconnaissant à Emmanuel Blanchard et les éditeurs de Plein droit de leur assistance pendant la rédaction de ce texte. 2 http://www.immigration.interieur.gouv.fr/Immigration/La-lutte-contre-l-immigrationirreguliere/Les-centres-de-retention-administrative-CRA p.221.
} 
police, ils préconisent « de s'en préoccuper d'urgence si l'on veut éviter qu'une masse de chômeurs, de malades, de sans-logis et d'asociaux ne se constitue sur le territoire métropolitain dès le prochain hiver. $»^{4}$

Faute d'un accord avec leurs homologues algériens, les autorités françaises introduisent de façon unilatérale des «contrôles sanitaires » à partir d'avril 1963. Tous les hommes algériens voyageant en quatrième classe, puis, lorsque le nombre de voyageurs en quatrième chute, tous les passagers arrivant pour la première fois dans le port de Marseille sont soumis à un examen médical. 600 personnes par mois en moyenne sont classées « inaptes » et l'entrée leur est refusée (environ $13 \%$ des personnes contrôlées). Par souci d'« efficacité » il est décidé que leur refoulement se fera par le premier bateau du lendemain, quelle que soit sa destination. Demeurait la difficulté de l'hébergement pendant une nuit. Le premier lieu choisi est un foyer du boulevard d'Anjou, derrière le port, mais le nombre de personnes «bloquées» ainsi que les difficultés de transport et de surveillance poussent les autorités à utiliser le local d'Arenc à partir de septembre 1963. À quelques centaines de mètres de la gare maritime il a aussi l'avantage d'être dans l'enceinte sécurisée de la zone portuaire.

S'agissant a priori de mesures sanitaires, cette dérogation aux accords d'Évian est justifiée par « l'intérêt public ». Mais les véritables intentions ressortent clairement d'un courrier du préfet des Bouches-du-Rhône au ministère de l'Intérieur de janvier 1964. Dans ses «propositions en vue d'enrayer le mouvement migratoire algérien », le préfet constate que les résultats du nouveau régime, « incontestablement satisfaisants du point de vue sanitaire, sont très modestes sur le plan de la migration et ne constituent [pas] un frein suffisant ${ }^{5}$ ». Cette analyse, partagée par de nombreux préfets des régions industrielles où sont installés la majorité des Algériens, établit un lien direct entre cette immigration et la prolifération des bidonvilles, et pointe la présence d'une «migration désordonnée dont les conséquences sont de plus en plus fâcheuses de tout point de vue ».

Cette vision, partagée par le ministère de l'intérieur, l'emporte au sein du gouvernement qui parvient à négocier une modification du régime de libre circulation avec l'Algérie. En avril 1964, l'accord Nekkache-Grandval introduit une carte de travail, délivrée préalablement en Algérie par l'ONAMO ${ }^{6}$

4 Rapport des CTAMs, troisième trimestre 1962, Préfecture des Bouches-du-Rhône, Archives des Bouches-du-Rhône (AbdR).

Rapport du préfet des Bouches-du-Rhône, 14 janvier 1964, envoyé aux ministère de l'Intérieur, ministère du Travail, ministère de la Santé Publique et de la Population, CAC19770391 article 5, Archives nationales, centre de Fontainebleau (ANF).

Office national algérien de la main-d'œuvre, préservant les apparences de la 
aux Algériens souhaitant travailler en France, à l'exception de certaines catégories (commerçants, étudiants). Le contingent de cartes accordées est fixé tous les trimestres par un comité mixte francoalgérien. Les ressortissants algériens peuvent toujours entrer en France sans la nouvelle carte ONAMO, mais ils sont alors soumis au régime «touriste» (séjour limité à trois mois sans autorisation de travail). L'accord comporte également une clause confidentielle qui prévoit une mesure expéditive de « rapatriement » :

Les ressortissants algériens qui, arrivés en France depuis plus de deux mois, n'ont pas trouvé d'emploi, ainsi que ceux qui, établis en France, se trouvent sans emploi ni ressources depuis plus de quatre mois, peuvent être rapatriés en Algérie par les soins du Gouvernement français, à moins que des raisons humanitaires n'y fassent obstacle ${ }^{7}$.

La circulaire du 17 avril 1964 définit l'application de cette clause : une décision préfectorale de rapatriement à la suite d'une enquête policière sur l'individu peut conduire à son éloignement sans recours possible. A Lyon et Marseille, où ce mécanisme est attendu avec impatience, les opérations dites de « rapatriement des oisifs » sont déclenchées immédiatement.

Ce durcissement de la réglementation concernant la population algérienne menace de surcharger le dispositif d'expulsion. Dès le printemps, le gouvernement français demande aux préfets de respecter des «créneaux d'horaires » pour le transfert des Algériens expulsés par arrêté ministériel à Marseille, les cellules du commissariat central arrivant à saturation « en raison du nombre allant croissant des Musulmans à refouler par bateau vers l'Algérie ${ }^{8}$. " Déjà surreprésentés parmi les étrangers frappés par des arrêtés ministériels, les Algériens sont, à la différence d'autres nationalités, régulièrement escortés à la frontière au moment de leur sortie de prison au milieu des années 1960.

À partir de mai 1964, le centre d'Arenc prend en charge, en plus des personnes non admises, tous ceux qui, sur le territoire de la métropole, sont expulsés par arrêté ministériel ou rapatriés par décision préfectorale. Les dortoirs sont aménagés, les différents groupes de détenus sont en principe séparés, et une compagnie du CRS est affectée à la surveillance du site sous l'autorité du commissaire Payan, officier des renseignements généraux et chef du commissariat spécial du port. Dans une lettre de juillet 1964, le ministère de l'Intérieur souligne que « les ressortissants algériens 7 8 op. cit, p. 214.

AbdR.
Archives diplomatiques du ministère des Affaires étrangères, cité par Alexis Spire, Circulaire ${ }^{\circ} 636$, ministère de l'Intérieur aux préfets, copie dans le fonds Payan, 
dont il s'agit n'étant pas sous le coup d'une peine privative de liberté, il importe que, durant leur séjour au centre, les mesures de surveillance soient strictement limitées aux dispositions nécessaires pour prévenir leur fuite ${ }^{9} »$. On ne saurait mieux résumer le caractère paradoxal d'une rétention administrative qui, de 1963 à 1975, a touché environ 50000 personnes.

Si le système d'escortes au commissariat spécial du port et d'enregistrement au centre de rétention dans l'attente du refoulement n'évolue guère au fil des années, la réglementation sur l'entrée et le séjour des étrangers est modifiée à plusieurs reprises, entraînant une augmentation et une évolution des caractéristiques de la population détenue. Le ministère de l'intérieur constate en effet qu'en l'absence d'une carte de séjour le régime «touriste » laisse la possibilité aux Algériens de s'installer durablement en France sans la carte ONAMO. La présence de ces « faux touristes algériens » guide une série de directives qui durcissent les critères d'entrée et créent des «quotas » mensuels de touristes. En mars 1965 une «notice individuelle pour touriste» est introduite qui fonctionne comme un visa, et d'autres instructions incitent les agents à la frontière à prendre en compte tout élément qui puisse « faire douter le but réel du voyage ». Le nombre de rapatriements explose : en mars 1965874 Algériens sont rapatriés par décision préfectorale (sur un total de 1571 détenus enregistrés au centre). La convergence de cette chasse aux «faux touristes » et de l'opération de « rapatriement des oisifs » explique le record d'expulsions de l'année 1965, plus de 11400 personnes ayant transité par Arenc. Devant les protestations du gouvernement algérien, le nombre de rapatriements baisse à partir de 1966, mais continue de toucher des familles entières.

En 1961, les autorités françaises avaient introduit un certificat d'hébergement pour les hommes algériens souhaitant être rejoints par leur famille. En théorie, il devait être établi au préalable par une mairie et attester que le logement pourrait accueillir tous les membres du foyer. Pendant plusieurs années ce fut une pure formalité, mais à partir de 1964, dans le contexte de la «lutte contre l'habitat insalubre », le ministère de l'intérieur exige des agents aux frontières une vérification plus stricte. Le chef du cabinet du préfet des Bouches-du-Rhône rappelle que les certificats «doivent être établis par les mairies et témoigner sans ambiguïté de la réalité d'un logement en France. Dans le cas contraire, les familles devront être conduites au centre $\mathrm{d}^{\prime}$ hébergement d'Arenc en vue de leur rapatriement ${ }^{10} »$. À Arenc, un dortoir accueille les femmes et les enfants bloqués à leur arrivée. En avril 1965, le préfet signale une modification du régime pour

9 10 juillet 1964, AbdR.

AbdR.
Chef du Cabinet du Ministre de l'Intérieur au préfet des Bouches-du-Rhône, 17

Note de service, Cabinet du préfet des Bouches-du-Rhône, 25 septembre 1964, 
les enfants de moins de 5 ans : «Les installations du centre d'hébergement d'Arenc ne permettant pas d'assurer aux jeunes enfants des conditions matérielles de séjour suffisantes, je vous recommande d'utiliser l'avion dans les cas précités ${ }^{11}$. »

Si l'immigration algérienne est la première visée, au cours des années 1960 certaines des pratiques s'élargissent à d'autres nationalités, essentiellement des ressortissants d'anciennes colonies françaises dont la présence en France est régie par des accords bilatéraux depuis l'indépendance. L'extension de mesures comme la notice individuelle de touriste conduit à l'augmentation du nombre de personnes non admises en provenance notamment du Maroc, de la Tunisie, du Mali et du Sénégal. Les nationaux de ces quatre pays, de plus en plus nombreux à être refoulés, représentent un peu plus de $10 \%$ des détenus en $1967,15 \%$ en 1968, et $30 \%$ en 1969, dans un contexte de diminution du nombre de refoulements de citoyens algériens. L'accord franco-algérien de 1968 instaure en effet une carte de résidence obligatoire pour les Algériens résidant en France, en contrepartie d'une limitation des rapatriements et de la fin de « quotas » pour l'entrée de touristes.

L'utilisation de la rétention à cette époque se distingue des pratiques actuelles sur deux points. $90 \%$ des personnes enregistrées au centre sont expulsées - contre moins d'une sur deux au cours des années 2000. Elles ne peuvent ni faire appel de la décision d'expulsion ni avoir accès aux conseils juridiques; les rares libérations de détenus interviennent au motif d'une hospitalisation ou parce qu'un officier du commissariat spécial du port reconnaît une erreur d'appréciation (dans les années 1960 on trouve dans les registres des commentaires tels que « libéré par ordre de l'OP - travaille à Marseille » ou « libéré - ancien harki »). Les modalités expéditives d'expulsion font que la durée de la rétention au centre est beaucoup plus courte qu'aujourd'hui, de 24 à 48 heures, et les cas de rétention de plus d'une semaine sont exceptionnels. Les conditions d'enfermement, sans aucun contrôle judiciaire ni suivi social, sont cependant très éprouvantes. Les registres conservent la trace d'hospitalisations à la suite de tentatives de suicides, de confrontations verbales et parfois physiques entre détenus et gardiens, et l'usage de cellules pour « calmer» les « excités ». En 1969, un groupe de détenus tente de lancer une grève de la faim contre les conditions de détention mais l'écho de cette action collective ne parvient pas à briser le mur de silence qui entoure Arenc. Dès le lendemain, quelques individus identifiés comme les «meneurs » sont embarqués d'office sur un navire ${ }^{12}$.

11 AbdR. 12
Lettre du préfet des Bouches-du-Rhône au commissaire Payan, 25 avril 1964, Registre de main courante 1969-1970, 2017 W 37, AbdR. 


\section{L'affaire d'Arenc}

Ce n'est qu'en 1975 que l'existence d'Arenc est dénoncée publiquement, entraînant un grand débat médiatique et politique. L'élément déclencheur est le cas de Mohamed Chérif, un Marocain travaillant à Marseille avec une carte de séjour valide. Un différend avec son consulat lui vaut d'être convoqué au commissariat central de Marseille le 11 avril 1975. Ses amis perdent ensuite sa trace et alertent maître Sixte Ugolini. Devant les réponses embarrassées et dilatoires de la préfecture, il convoque une conférence de presse, le 16 avril, afin de dénoncer la disparition de son client. Le soir même, sur ordre de la préfecture, Chérif est libéré alors qu'il allait être embarqué pour le Maroc depuis le port de Sète, et raconte à Sixte Ugolini sa rétention à Arenc.

La presse locale, suivie par une partie de la presse nationale, s'empare de l'affaire. Le 22 avril, France 3 y consacre un reportage alimenté par les communiqués du Syndicat des avocats de France. Une semaine après, à l'Assemblée nationale, le député communiste Paul Cermolacce et le maire de Marseille, Gaston Defferre, exigent des explications. Le gouvernement argue qu'Arenc est un simple «centre d'hébergement» fonctionnant en toute légalité. Paul Dijoud, secrétaire d'Etat en charge des travailleurs immigrés, assure à la télévision qu'il n'y a pas de « prison clandestine ».

D'anciens détenus se manifestent alors et deux d'entre eux portent plainte contre $\mathrm{X}$ pour séquestration : Salah Berrebouh, un jeune Algérien, expulsé alors qu'il était sous contrôle judiciaire et revenu à Marseille, et Gustave Essaka, un Camerounais détenu à Arenc pendant deux semaines en 1971 et libéré après un aller-retour à Alger où son débarquement a été refusé. Saisi de l'affaire, le juge Loques effectue une perquisition à Arenc le 29 mai. La pression est maintenue pendant l'été par des manifestations - dont une marche au port de Marseille en juin qui rassemble plusieurs centaines de personnes selon la préfecture. À la rentrée, le journaliste Alex Panzani publie Une prison clandestine de la police française : Arenc.

La mise en cause de hauts fonctionnaires ralentit le déroulement de l'enquête. Baladé entre différentes instances, le dossier du juge Loques n'aboutit pas et, en 1978, les plaignants sont déboutés. Mais si l'administration n'est pas sanctionnée, le gouvernement rencontre plus de résistance lorsqu'il tente de donner une base légale à la détention pratiquée à Arenc. La circulaire du 21 novembre 1977, vivement critiquée par le Syndicat de la magistrature et le Syndicat des avocats de France, incarne ce bricolage réglementaire. Elle est annulée le 7 juillet 1978 par un arrêt du Conseil d'État, mais un décret du 9 décembre 1978 reprend l'essentiel du texte tout en substituant une durée « limitée au temps strictement nécessaire à l'exécution effective de l'expulsion » aux 7 
jours de rétention initialement prévus. Depuis janvier 1978, l'administration avait suspendu la rétention à Arenc des personnes en cours d'expulsion, les commissariats et les prisons assurant l'intérim. De 1978 à l'entrée en vigueur de la loi du 29 octobre 1981 ${ }^{13}$, Arenc « héberge » uniquement les personnes non admises. La loi Bonnet de janvier $1980^{14}$, censée fixer un cadre légal à la rétention, suscite de nombreuses protestations à l'Assemblée et au Sénat. Les critiques transcendent largement les clivages partisans : des députés et sénateurs de droite mêlent leurs voix à celles de la gauche et ce n'est qu'avec d'importants amendements que le gouvernement parvient à faire adopter son texte ${ }^{15}$. Le 29 octobre 1981, Gaston Defferre, devenu ministre de l'intérieur, donne son nom à un nouveau texte de loi qui instaure des garanties supplémentaires en termes d'accès au conseil juridique, de droit de recours, de catégories protégées de l'expulsion et de conditions de détention. Mais le principe de la rétention administrative des étrangers est entériné comme outil légitime de la politique d'immigration. Malgré de nombreuses promesses sur la démolition d'Arenc dont les conditions matérielles indignes sont l'objet de critiques répétées au fil des années - le hangar est officialisé et fera partie du réseau national des centres de rétention administrative jusqu'à sa fermeture définitive en 2006.

La dénonciation de la «prison clandestine» d'Arenc a ainsi conduit à la légalisation et à la banalisation progressive de la rétention administrative. Mais la période 1975-1981 a cependant marqué une rupture avec une décennie pendant laquelle environ 50000 personnes ont fait l'expérience d'une détention hors de tout contrôle judiciaire dans un local soustrait aux regards externes. Panzani évoquait dans son livre «une prison où les policiers sont à la fois juges et gardiens ». Vues sous cet angle, la campagne de 1975 voire la loi du 29 octobre 1981, peuvent être considérées comme une victoire fondatrice, certes très incomplète, du combat par le droit.

13

en France

14 15
Loi n 81-973 du 29 octobre 1981 relative aux conditions d'entrée et de séjour des étrangers Loi n ${ }^{\circ} 80-9$ du 10 janvier 1980 relative à la prévention de l'immigration clandestine. Alexis Spire, « Rétention : une indignation oubliée », Plein droit, n50, juillet 2001 http://www.gisti.org/spip.php?article4152 